\title{
Articles You May Have Missed
}

\author{
Robert Goodnough $^{1}$ (D) C Charles Edward Murphy IV ${ }^{1} \cdot$ Craig Smollin $^{1}$ • \\ Daniel Repplinger ${ }^{1}$
}

Received: 30 August 2017 / Revised: 10 September 2017 / Accepted: 11 September 2017 / Published online: 24 January 2018

(C) American College of Medical Toxicology 2018

Keywords Chironex fleckeri (box jellyfish) - Salicylate . Hemodialysis · Carbon monoxide · Hyperbaric oxygen · Envenomation $\cdot$ Varespladib $\cdot$ Phospholipase A2 inhibitor

\section{Article Title}

Isbister GK, Palmer DJ, Weir RL, Currie BJ: Hot water immersion $\mathrm{v}$ icepacks for treating the pain of Chironex fleckeri stings: a randomized control trial. Medical Journal of Australia 2017;206(6):258-261.

\section{Background}

The current standard of care for Chironex fleckeri in northern Australia involves application of vinegar, management of life-threatening effects, and icepacks for pain. If icepacks fail to adequately relieve pain, oral or parenteral analgesias are recommended. Heat immersion therapy has not been previously investigated for management of pain due to C. fleckeri stings.

\section{Research Question}

Is hot water immersion at $45^{\circ} \mathrm{C}$ superior to icepack application for reducing pain due to $C$. fleckeri stings?

\section{Robert Goodnough}

rtgoodno@gmail.com

1 University of California San Francisco, San Francisco, CA, USA

\section{Methods}

Single-center, open-label, randomized controlled trial comparing hot water immersion at $45{ }^{\circ} \mathrm{C}$ with icepack application for treatment of pain due to $C$. fleckeri stings. Patients age $\geq 8$ years presenting to the emergency department (ED) within $4 \mathrm{~h}$ of suspected $C$. fleckeri stings and without evidence of severe systemic or ocular effects were enrolled. All patients had vinegar applied to their wounds prior to initiation of hot water immersion or icepack therapy. The primary outcome measure was pain severity at $30 \mathrm{~min}$ post-treatment, as assessed by a visual analog scale. The primary outcome was assessed by intention-to-treat analysis. Secondary outcomes included ED length of stay (LOS), need for opioid analgesia, crossover to alternative treatment, and delayed urticaria.

\section{Results}

Forty-two patients were randomized to icepack $(n=25)$ or hot water immersion $(n=17)$ therapy. Demographics and severity of envenomation were similar between groups. All were discharged from the ED; 95\% of patients were pain free at discharge. Clinically meaningful pain improvement at 30 min was achieved in $56 \%$ of patients treated with ice and in $65 \%$ treated with hot water (absolute difference $9 \% ; 95 \% \mathrm{CI}-22$ to $39 \% ; p=0.75$ ). The LOS was $1.6 \mathrm{~h}$ for icepack treatment (IQR $1-1.8 \mathrm{~h}$ ) and $2.1 \mathrm{~h}$ for hot water treatment (IQR $1.6-1.8 \mathrm{~h} ; p=0.07$ ). One patient from the icepack group crossed over to hot water immersion and two patients from each group required intravenous opioids. No patients re-presented with recurrent pain. 


\section{Conclusion}

Hot water immersion at $45{ }^{\circ} \mathrm{C}$ was no more effective than icepacks for treating pain due to C. fleckeri stings but is more labor intensive and may increase LOS. After application of vinegar to the sting site, icepacks remain the recommended ED treatment for pain due to significant box jellyfish stings.

\section{Critique}

The study was underpowered and groups were unequally allocated due to a smaller than expected treatment effect and due to the block size used in the blocked randomization. Although the authors concluded that LOS was longer for heat immersion, the difference was not statistically significant $(p>0.05)$. Though heat immersion was not found to be superior to icepack, it was as effective for pain control. The treatment effect of hot water immersion may have been limited due to a delay between envenomation and initiation of therapy.

\section{Implication for Toxicologists}

The data do not support changing current protocols recommending icepacks for pain reduction of box jellyfish stings. Future research should investigate the initiation of hot water immersion in the prehospital setting. Hot water immersion as a second-line therapy, prior to initiation of opioid analgesia, should be investigated.

\section{Article Title}

McCabe DJ, Lu JJ: The association of hemodialysis and survival in intubated salicylate-poisoned patients. American Journal of Emergency Medicine 2017; https://doi.org/10. 1016/j.ajem.2017.04.017.

\section{Background}

Salicylate (ASA) poisonings are common but varied presentation and severity lead to inconsistent treatment. In severe poisoning, mental status changes or fatigue of respiratory compensation may require endotracheal intubation. Mechanically ventilated patients risk inadequate respiratory compensation for acidosis and worsening ASA poisoning. Hemodialysis (HD) is inconsistently applied to these patients.

\section{Research Question}

Is there an association between survival and HD in ASApoisoned patients who require endotracheal intubation?

\section{Methods}

Retrospective, observational review of ASA poisonings called to the Illinois Poison Center between 1/1/2003 and 12/31/ 2014. All intubated patients with serum ASA levels $>50 \mathrm{mg} / \mathrm{dl}$ were included.

\section{Results}

A total of 56 cases were identified. Overall survival was $73.2 \%$, and $73.5 \%$ (11/15) of fatal cases did not receive HD. For cases that did not receive HD, an ASA level of $>50 \mathrm{mg} / \mathrm{dl}$ had a survival rate of $56 \%(14 / 25)$, compared to $0 \%(0 / 9)$ with levels $>80 \mathrm{mg} / \mathrm{dl}$. In patients who received HD, an ASA level $>50 \mathrm{mg} / \mathrm{dl}$ had a survival rate of $83.9 \%$ (26/31), compared to $83.3 \%(15 / 18)$ of those with a level $>80 \mathrm{mg} / \mathrm{dl}$. All 31 cases that received HD were previously intubated. Among the 9 cases with an initial ASA level $<50 \mathrm{mg} / \mathrm{dl}$ but subsequent level $>50 \mathrm{mg} / \mathrm{dl}$, HD did not affect survival.

\section{Conclusion}

In patients with ASA levels $>50 \mathrm{mg} / \mathrm{dl}$ (particularly those with levels $>80 \mathrm{mg} / \mathrm{dl}$ ) who required endotracheal intubation, HD was associated with increased survival.

\section{Critique}

The authors did not include 6 patients who received incomplete HD (due to cardiac arrest) in the HD group, which likely skewed results. Retrospective data was limited; it is possible that patients not receiving HD were too unstable for this intervention. As a poison center-based study, limited patient information (e.g., pre-existing conditions, co-ingestions, relevant laboratory data [e.g., serum $\mathrm{pH}]$ ) were not available, therefore introducing numerous confounders. Lastly, the small sample size underpowered the ability to detect a survival difference.

\section{Implication for Toxicologists}

These data do not support lowering the threshold for HD in intubated patients with ASA toxicity. However, the apparent 
higher mortality among non-dialyzed, intubated patients indicates that further investigation is warranted.

\section{Article Title}

Huang CC, Ho CH, Chen YC, Lin HJ, Hsu CC, Wang JJ, Su SB, Guo HR: Hyperbaric oxygen therapy is associated with lower short- and long-term mortality in patients with carbon monoxide poisoning. Chest 2017; doi: 10.1016/j.chest.2017.03.049.

\section{Background}

The effect of hyperbaric oxygen therapy (HBOT) on mortality in patients with acute carbon monoxide $(\mathrm{CO})$ poisoning is controversial, and which patients may benefit from HBOT remains unknown.

\section{Research Question}

Does HBOT reduce mortality in patients with $\mathrm{CO}$ poisoning?

\section{Methods}

Retrospective cohort study of 25,737 patients diagnosed with CO poisoning in Taiwan between 1999 and 2013. The mortality risk of patients who received HBOT was compared with those who did not receive HBOT. Multiple stratified subgroup analyses were performed based on age, gender, comorbidities, acute organ failure, suicide attempt, and income. Data were obtained via ICD-9 code searching of the Nationwide Poisoning Database, which is a sub-database of the Taiwan National Health Insurance Database. Mortality risks of the cohorts were compared with Cox proportional hazard regression adjusted for age, sex, and numerous medical and socioeconomic comorbidities.

\section{Results}

Patients who received HBOT had lower mortality risk, with an adjusted hazard ratio (AHR) of 0.74 (95\% CI 0.67-0.81). Stratified analysis showed this was true for all age groups (particularly age $<20$ years; AHR 0.45 ; 95\% CI $0.26-0.80$ ), gender, suicide attempts, drug poisoning, and comorbidities (hypertension, hyperlipidemia, liver disease, and mental health disorder). HBOT reduced mortality in patients with and without acute respiratory failure (AHR $0.43 ; 95 \%$ CI $0.35-0.53$ and AHR $0.84 ; 95 \%$ CI $0.76-0.93$, respectively). HBOT was associated with reduced mortality at all follow-up points ( 2 weeks to
4 years). A subgroup analysis demonstrated reduced mortality for patients receiving $\geq 2$ HBOTs, compared to only one session. However, $>5$ sessions were associated with intermediate long-term survival benefit, suggesting against a dose response.

\section{Conclusion}

HBOT was associated with reduced mortality risk in patients with $\mathrm{CO}$ poisoning, particularly among younger $(<20$ years $)$ patients and those with acute respiratory failure.

\section{Critique}

There were numerous limitations that hinder clinically useful conclusions. It was a retrospective review of limited data involving patients without randomization of treatment. No clinically relevant data (CO levels, physical examinations, vital signs, evidence of end-organ dysfunction, co-ingestions) were provided or controlled for. Compared with the non-treatment group, patients receiving HBOT were younger (including fewer $>65$ years) and had fewer comorbidities. Patients with acute respiratory failure were identified based on diagnosis codes and need for intubation/mechanical ventilation; thus, it is unknown if the etiology of respiratory failure was similar between groups. The cause of death for these patients was not reported and may not have been sequelae of $\mathrm{CO}$ poisoning. The treatment protocols for HBOT were not standardized, and all HBOT initiated within 1 month of $\mathrm{CO}$ poisoning was included. The authors' definition of mortality included patients who left against medical advice or left the national healthcare system. Why such patients were counted as dead was not explained.

\section{Implication for Toxicologists}

The results of this study should be interpreted with caution due to the possibility of selection bias, which may lead to an overestimation of the treatment effect.

\section{Article Title}

Lewin M, Samuel S, Merkel J, Bickler P: Varespladib (LY315920) appears to be a potent, broad-spectrum, inhibitor of snake venom phospholipase A2 and a possible pre-referral treatment for envenomation. Toxins 2016;8(248);doi:10.3390/ toxins 8090248 . 


\section{Background}

Snake envenomation results in 125,000 annual deaths worldwide, with an estimated $75 \%$ of these deaths occurring prehospital. Broad-spectrum inhibitors of snake venom have the theoretical promise of providing point of care treatment. Two such potential drugs are varespladib and its prodrug methyl-varespladib, which are secretory phospholipase A2 (PLA2) inhibitors.

\section{Research Questions}

Do varespladib and methyl-varespladib provide PLA2 inhibition in snake venom across multiple species in vitro; and does intravenous (IV) varespladib provide survival benefit in rodents inoculated with lethal doses of snake venom?

\section{Methods}

\section{Part 1}

Descriptive study of in vitro chromogenic assays to plot doseresponse curves for varespladib and methyl-varespladib inhibition of PLA2 from bee venom (control) and from venoms of 28 snake species.

\section{Part 2}

Prospective proof of concept in vivo survival studies involving envenomated rodents treated with varespladib. Model one compared 24-h survival of five mice, pre-treated with varespladib, compared to controls. Both groups were injected with lethal doses of elapid venom. Model two compared survival of concurrent or post-venom varespladib treatment, versus control, in mice injected with lethal doses of Vipera berus venom. Model three compared survival, PLA2 activity, and hemolysis in three groups of rats treated with 4 or $8 \mathrm{mg} / \mathrm{kg}$ doses of M.fulvius venom: group 1 was controls, group 2 received varespladib at $30 \mathrm{~s}$ after venom injection, and group 3 received varespladib $5 \mathrm{~min}$ after injection of venom.

\section{Results}

Varespladib and methyl-varespladib had high-potency in vitro inhibition of PLA2 activity in venom from 28 species of snakes. Mouse model one showed $100 \%$ death in control mice at $63 \mathrm{~min}$, compared with varespladib-treated mice surviving between 8 and $30 \mathrm{~h}$. Model two showed survival benefit of varespladib (IV more so than subcutaneous [SQ]), compared to controls, at $24 \mathrm{~h}$ for animals receiving varespladib with or after (unknown time interval) $V$. berus venom injection. Model three showed greater survival $(100 \%)$ at $24 \mathrm{~h}$ in rats injected with IV varespladib (5 min after lethal doses of venom administration) compared to controls. Model three also showed inhibition of PLA2 activity and hemolysis in varespladib-treated rats compared to controls.

\section{Conclusion}

Varespladib and methyl-varespladib showed highly potent in vitro activity against PLA2 in 28 snake venoms. In rodents, pre-treatment, concurrent treatment, and rescue treatment with IV or SQ varespladib provided survival benefit up to $24 \mathrm{~h}$ after lethal dose envenomation from three different snake species.

\section{Critique}

Extracting human relevance from animal data is difficult, and the benefit appears limited to parenteral administration of varespladib prior to, concurrent with, or within $5 \mathrm{~min}$ of envenomation. And these effects are limited to $\sim 30 \mathrm{~h}$. The authors readily acknowledged these limitations. A potential conflict exists due to the primary investigator owning a company (Ophirex® Inc.) which markets snakebite antidotes.

\section{Implication for Toxicologists}

These data suggest that broad-spectrum inhibitor therapy (in the prehospital setting) for snakebite may be a bridge to antivenom and comprehensive care. More extensive in vivo testing, across venom types, and the development of an oral formulation are needed. The benefit of these therapies requires optimized timing and dosing of administration for greatest benefit.

Sources of Funding None

\section{Compliance with Ethical Standards}

Conflicts of Interest The authors declare that they have no conflicts of interest. 